\title{
Development of a student-centered instrument to assess middle school students' conceptual understanding of sound
}

\author{
Haim Eshach \\ Ben Gurion University of the Negev, Beer Sheva 8410501, Israel
}

(Received 10 March 2013; published 21 January 2014)

\begin{abstract}
This article describes the development and field test of the Sound Concept Inventory Instrument (SCII), designed to measure middle school students' concepts of sound. The instrument was designed based on known students' difficulties in understanding sound and the history of science related to sound and focuses on two main aspects of sound: sound has material properties, and sound has process properties. The final SCII consists of 71 statements that respondents rate as either true or false and also indicate their confidence on a five-point scale. Administration to 355 middle school students resulted in a Cronbach alpha of 0.906, suggesting a high reliability. In addition, the average percentage of students' answers to statements that associate sound with material properties is significantly higher than the average percentage of statements associating sound with process properties $(p<0.001)$. The SCII is a valid and reliable tool that can be used to determine students' conceptions of sound.
\end{abstract}

DOI: 10.1103/PhysRevSTPER.10.010102

PACS numbers: 01.40.- d, 43.10.Sv

\section{INTRODUCTION}

The goal of this paper is to describe the development of a student-centered instrument aiming at assessing students' conceptual understanding of sound. I will first provide some background, such as the need for the development of such an instrument, and the differences between the traditional convergent-type assessment tool and the student centered one presented here. I will then describe the data sources used for developing the instrument, namely, students' concepts of sound and historical concepts of sound. After this background, I will describe the development of the instrument itself, how the objectives or themes of the instrument were determined, the form of the questionnaire, how the items were developed, and how the instrument was tested.

\section{STANDARDIZED ASSESSMENT TESTS: AN OVERVIEW}

\section{A. The need for an assessment instrument in sound}

\section{Why are standardized tests important?}

The present study joins the long term interest amongst science education researchers in creating valid, standardized, meaningful, and practical instruments for assessing school students' understanding of concepts in physics; in this case, the concept of sound. Efforts to develop assessment tools have been made in a variety of physics domains,

Published by the American Physical Society under the terms of the Creative Commons Attribution 3.0 License. Further distribution of this work must maintain attribution to the author(s) and the published articles title, journal citation, and DOI. such as mechanics (e.g. the Force Concept Inventory [1], the Mechanics Baseline Test [2], and the Test of Understanding Graphs in Kinematics [3]), astronomy [4], optics [5], and electromagnetics [6]. Assessment tools may help teachers to identify difficulties and barriers faced by their students in understanding scientific phenomena, as Vosniadou et al.'s [6] (p. 392) following quotation notes:

"The realization that students do not come to school as empty vessels but have representations, beliefs, and presuppositions about the way the physical world operates that are difficult to change has important implications for the design of science instruction. Teachers need to be informed about how students see the physical world and learn to take their points of view into consideration when they design instruction. Instructional interventions need to be designed to make students aware of their implicit representations, as well as of the beliefs and presuppositions that constrain them."

Identifying students' misconceptions is therefore a crucial step in the teaching process that may enable teachers to design effective learning environments that help reshape students' initial knowledge into scientifically accepted understanding [5,7]. Indeed, according to different theories of conceptual change, during the teaching process we may want to address students' existing spontaneous reasoning, replace it, reorganize it, or refine and build on it [8].

Support for the importance of the need for valid standardized assessment tools can be found in other domains of science education as well. For instance, in making the point for the importance of developing a valid standardized assessment tool for examining students' views of the nature of science (NOS), researchers claim that such 
assessment tools may enable NOS to be included in largescale assessments at the state or national level [9]. Doing so, according to Chen [10], might change the current situation, in which NOS does not receive the requisite attention it ought to in today's schools. Developing an assessment instrument for the concept of sound might make similar changes in the attention it receives from schools, which also does not currently meet its requirements [11].

Such an instrument would also allow science educators and teachers to conduct comparison studies and relate students' conceptual understanding of sound to other measurable educational outcomes. In addition, although the present study aims at developing an assessment tool for middle school students, it might be useful in assessing the perception of middle school science teachers as well (for other instances in which this has proven to be the case, see Trumper [12]).

\section{Why sound?}

If one goal of science education is advancing students' learning about their own world and about "useful knowledge" (see Layton [13], especially chap. 5), then the subject of sound should be an essential component of science curricula. For students to understand theoretical and practical questions, such as whether one can hear sound in water, why it is dangerous to listen to loud music, how a physician's stethoscope works, or how ultrasound works, they require a firm foundation in physical science concepts connected with sound [11]. Also, according to Hrepic et al. [7], because sound is a wave phenomenon, its understanding may contribute significantly to understanding both classical and modern physics. Thus, identifying the preconceptions that prevent students from properly understanding sound phenomena deserves a central place within the science curriculum and should also be addressed in science education research.

\section{B. Assessment instruments: traditional convergent-type vs student-centered}

In recent years, several objections have been raised in relation to the use of convergent-type instruments to assess learners' conceptions. In such instruments students must choose between predefined answers, which reflect the ideas that the instrument's developers believe students might have, thus preventing students from providing answers of their own that express their true perceptions. For example, in the case of the use of multiple-choice instruments for assessing students' views concerning NOS, many researchers have argued that by forcing a choice between given answers, convergent-type instruments could impose the instrument developers' conceptions of NOS on respondents [14-15], which would threaten these instruments' validity [16]. Student centered instruments seek to address this concern. In such instruments, students are asked to provide their own answers.
Instruments of this sort include in-depth interviews, which have proven to be extremely fruitful [3,17-18] and open-ended questionnaires [15]. However, interviews and open-ended instruments are not immune to criticism either. Indeed, researchers have detailed several weaknesses of open-ended tools. For instance, Chen [10] argues that it is challenging for participants to fully articulate their views in 40-60 minutes, and it is difficult for researchers to gain the intended information from every participant. Indeed, in my own experience, whenever students are asked to fill open-ended questionnaires, they tend to use short laconic answers that do not necessarily clarify their understanding of the topic. Furthermore, open-ended questions require a much higher level of writing ability, so that the tests may measure writing ability more than they do understanding of a topic. It is not surprising therefore that Lederman's group themselves [18] suggest conducting follow-up interviews to clarify whether what was written in the open questionnaire that they developed indeed reflects students' views. The follow-up interviews, according to these authors, provide students the opportunity to further elaborate on and articulate their answers and views.

Interviews, open-ended questionnaires, or a combination of the two all share an additional difficulty—namely, that they are practically untenable in a large sample of students because of the enormous resources necessary for such an undertaking-resources that are usually not available to researchers [19]. Furthermore, according to Beichner [3] "the ability to statistically analyze data from large numbers of objectively graded multiple choice exams may allow greater generalizability of the findings, albeit with lower resolution than interview-based results" (p. 750). In summary, it seems that, on the one hand, open-ended questionnaires and especially interviews - or a combination of both-can have greater resolution than multiple-choice tests, but, on the other hand, these tools are limited in cases where large-scale assessment is needed.

One course of action offered by researchers that may provide some solution to the above concern is to use a student-centered process to construct the instrument items as follows: (a) using known students' misconceptions as distracters in questionnaires that aim at identifying students' difficulties (e.g. [20-21]). Instead of basing its conception of students' possible viewpoints on the postulations of education theory of research, this approach bases its assumptions on empirical records of false perceptions students are known to have had. This basis may bring an unusually high degree of validity to the instrument's distracters, because the meaning that students read into the instrument choices tends to be the same meaning that students would express if they were spontaneously verbalizing their own views in an interview [22]. (b) Allow students, in an open-ended format, to articulate whatever ideas they deem representative of their viewpoint on the target issue. For this purpose, the following choice should 
be added to each of the questionnaire's questions: "None of the above choices fits my basic viewpoint. My basic viewpoint is (please explain your viewpoint in the space provided below)" [19].

\section{Selecting data sources}

The development of a student-centered instrument aiming at assessing students' conceptual understanding of sound must take into account the elements in comprehending sound that students find most difficult. To this end, we made use of two main data sources in the development of the instrument process: known students' difficulties in understanding sound and the history of science. The history of science can be a useful data source because some of the common misconceptions about scientific concepts (including sound) that may be found in historical texts correspond to similar misconceptions in modern students. The misconceptions of historical figures can therefore be used to identify similar misconceptions amongst students $[11,23]$. A brief description of the various ways in which sound had been (mis)understood will therefore be provided.

But before presenting students' difficulties in understanding sound and briefly describing its historical development, it is worthwhile to first describe what ninth grade students are expected to know about sound (in this case, based on their progression through the uniform national Israeli science curriculum). Students at this age should know that (a) sound needs a material medium; it cannot be created in a vacuum; (b) sound is related to its medium's pressure change; and (c) sound can propagate not only through air but also through liquids such as water and through solids. The scientific view, of course, does not perceive sound as a kind of matter, although it requires matter (as a medium). A deep understanding of sound should therefore include the understanding that sound itself is not a kind of matter, but rather that it is a material phenomenon.

\section{Students' concepts of sound}

Naive conceptions of sound have not been studied extensively [24]. Boyes and Stanisstreet [25] examined middle school students' conceptions of the path of sound between a source and a listener. The subjects were presented with a picture of a radio and one of a person. They were asked to answer the question, "How do you think sound travels, enabling the person to hear what is on the radio?" and to draw arrows on the picture indicating the direction of the sound path. The results showed that only about $40 \%$ of the younger pupils (ages 11-13) and $78 \%$ of the older group (ages 13-16) indicated that sound travels from the source to the hearer. Asoko et al. [26] investigated pupils between the ages of 4 and 16, and found that younger children often link the production of sound with their own actions, or consider sound to be a part of the object from which it originated. In addition, Asoko et al. found that the notion of sound traveling within the air existed only amongst 16 year olds (having a $70 \%$ reference within this group).

Chi and colleagues [27-29] suggest that students' misconceptions are attributable to a mismatch between the ontological categories to which subjects assign concepts and the ontological categories to which concepts usually belong. In physics, for example, subjects have trouble understanding concepts such as electrical current, heat, and light because they assign these entities to the category of "matter" when they belong to the ontological category of "processes" [30]. Chi et al. state that "novices seem inclined toward materialistic or substance-based conceptions" (p. 2) when it comes to describing abstract physics concepts. For instance, students may perceive electric current as a kind of "electricity juice" or "electron juice," which is the current itself and flows from one end of a wire to the other, rather than viewing electric current as a process involving the entire circuit, including material elements such as electrons. This was found to be valid for the case of sound too [24].

Several studies have identified a tendency towards materialistic perceptions of sound amongst middle school students. For instance, a study described by Driver et al. [31] is that of Watt and Russell [32], which suggests that middle school students may envisage sound as an invisible object with dimensions, which requires space in order to move. Eshach and Schwartz [11] used Reiner et al.'s substance schema - properties that are common to material substances and that may be extended generally to describe any material substance - to examine whether middle school students possess materialistic thinking of sound. Consistent with the substance schema, sound was perceived by the participants as being pushable, frictional, containable, or transitional. All in all, it seems that sound, which is a common phenomenon that we experience every day, is an area in which students display numerous difficulties in understanding.

\section{E. Historical conceptions of sound}

Connections between historical perceptions of scientific concepts and students' views of these concepts have been documented in the literature. Chi [27], for instance, claims that "there is an explicit similarity in the conceptions of physical science concepts by naïve students and medieval scientists, and I interpret this correspondence to arise from both groups of people having adopted a material substance view" (p. 159). The following quote from Lucretius, a Greek philosopher from 100 B.C., illustrates the observation underlying Chi's claim:

"The fact that voices and other sounds can impinge on the senses is itself a proof of their corporeal nature. Besides, the voice often scrapes the throat and a shout roughens the windpipe on its outward path. What happens is that, when atoms of voice in greater numbers than usual have begun to 
squeeze out through the narrow outlet, the doorway of the overcrowded mouth gets scraped. Undoubtedly, if voice and words have this power of causing pain, they must consist of corporeal particles" [33] (pp. 146-147).

Since historical ideas of a scientific concept can also be found in students' views of this same concept [11], it is argued herein that historical material can be used while developing an instrument that aims to examine students' views concerning this concept. It is important to stress in this context that ideas taken from history were incorporated into the questionnaire only after a thorough literature review had made it clear that similarly material perceptions of sound-ideas that correlate to the historical ones-had been identified in modern students. The historical conceptions thus merely served to provide detailed content for plausible distracters, not as independent suggestions for possible misconceptions.

\section{DEVELOPMENT OF THE SOUND CONCEPT INVENTORY INSTRUMENT (SCII)}

\section{A. Determining the SCII objectives or themes}

According to Beichner [3], once it is established that developing a questionnaire is a worthwhile effort, it is necessary to formulate a list of specific objectives that relate to an understanding of the concept at which the questionnaire is aimed. Based on the literature review, in interviews with five high school physics teachers, three physics professors, and two physics education professors, the following two main objectives were identified:

1. Students at the middle school level should understand that sound is a process phenomenon characterized by the following

(a) sound is connected to movement of the medium's particles;

(b) sound relates to changes in the medium's pressure or density.

2. Students should understand that sound does not possess material characteristics. The following list of material characteristics, which students must understand sound not to have, is based on the work of Reiner et al. [30] and Eshach and Schwartz [11].

(a) invisible material;

(b) having a corpuscular nature-having surface and volume;

(c) being pushable — able to push and be pushedI. by objects, and II. by the medium;

(d) being frictional-experiencing "drag" when moving in contact with some surface;

(e) being containable - able to be contained by something;

(f) being consumable - capable of being depleted;

(g) being gravity sensitive-falls down when dropped; (h) hearing being influenced by the sound particle's size or number;

(i) being able to pass in a vacuum-according to Hrepic et al.'s [7] view that sound propagates through the vacuum indicates that sound is viewed as an independent kind of material.

This list of material characteristics of sound is based on the "substance scheme," namely, those properties that are common to material substances and which may be extended generally to describe any material substance [30]. Eshach and Schwartz [11] recommended that in the case of sound Reiner et al.'s substance scheme would have to be revised. The list above is therefore a more specifically adapted substance scheme for sound.

\section{B. Deciding the questionnaire's form}

Usually assessment instruments that examine students' conceptual understanding of a scientific concept are multiple-choice questionnaires, in which students must choose from amongst several statements the one that they believe best explains a problem. While this form of questionnaire would have been easily applicable here, I chose another format, in which the students are requiredinstead of choosing just one item-to respond to all of them, stating for each whether it is correct or not. Roedel et al. [34] argue that by utilizing a mechanism that demands the reader to choose more than only one correct answer, researchers can better pinpoint students' level of understanding of a particular concept. In the same manner, the instrument presented in this paper enables the students to choose more than one correct or incorrect statement. This format is particularly well suited to identifying situations in which students hold views of sound as being associated both with processes (e.g. changes in pressure or density of the medium) and with matter (e.g. perceiving sound as "pushable").

The students were also asked to decide the level of their confidence in their answer on a 5 point scale, and only the answers for which the students' confidence was 4 or higher were used in the data analysis. The decision to address only the data from the higher certainty levels was made-in consultation with experts-in order to tighten the data analysis. This, I believe, is especially important while developing a new instrument. Adding the reference to their certainty level allows us to address problems that arise from the possibility that the students are guessing. If a participant marked a particular statement as true, but marked a very low certainty level, we can suppose this choice to have been a guess, and address it appropriately. In this respect, the questionnaire differs from standard multiple choice questionnaires, which do not distinguish between questions that participants are sure about and questions to which they have guessed the answers.

Finally, in addition to the predefined statements in each question, the students were also provided with the 
following option: "None of the above choices fits my basic viewpoint. My basic viewpoint is (please explain your viewpoint in the space provided below)". All of the students' open-ended answers were read and reread by the author and one physics teacher separately. After reading the answers the author and the physics teacher decided whether indeed the student had provided an answer that does not exist in the predefined statement, or whether the student's answer could be associated with one of the predefined statements, expressed in different words but conveying the same meaning. Since only a few students used the option of providing their own answers, the whole process took about 3 hours for each reader, after which the author and the physics teacher met together to discuss their decisions on each open answer. This process took about 2 hours. Of these open answers, 20\% included statements such as, "I do not know the answer" or "I have no idea." In this case, the "no" option in the questionnaire was chosen for that question in the SPSS file. The rest of the students' answers (80\%) were in fact differently worded expressions of an idea presented in the predefined statements. These statements were input to the analysis.

\section{Determining the assessment tool's items}

\section{Using students' difficulties}

As was noted earlier, one solution to the concern that multiple-choice questionnaires may not reflect students' own concepts but rather those of the instrument developers is to use students' own ideas as distractors. Question 13 in the questionnaire (see Appendix), taken from Eshach and Schwartz [11], where interviews with middle school students concerning a variety of sound phenomena were conducted, is such an example. Distractors $a, b, d$, and $e$ were provided by the students in that study (i.e. they were taken from their interviews). The idea of gravity (noted by students and used in distractor $b$, question 13) was also used in question 16 in the questionnaire (see Appendix).

\section{Using historical materials}

Here are two examples demonstrating the use of historical material for the purpose of this instrument. In developing question 12 (see Appendix), I was inspired by Lucretius's view of sound (see theoretical background section). Distractors $a$ and $b$ of the question reflect the idea that sounds are corpuscular particles that can have the power to scrape the throat. Distractor $a$ reflects the exact meaning in the quotation while distractor $b$ reflects an idea students expressed in my previous study [11]. The historically based distractor $a$ provides an additional possibility for the students to choose from, while still clearly reflecting a very similar, material view of sound to that based on the perceptions of modern students (i.e. distractor $b$ ). Question $8 \mathrm{f}$ in the questionnaire (see Appendix) reflects Isaac
Beeckman's (1588-1637) view of sound: when we speak, the sound coming out of our mouths is carried in invisible bubbles. These bubbles are pushed by the air, and when they reach the hearer's ears, the sound exits the bubbles and enters the ears.

Isaac Beeckman supposed that sound travels through the air as globules of sonic data. He considered that every vibrating object cuts the air around it into small spherical corpuscles. Those corpuscles are forced to travel in the air in all directions by the motion of the source and are obtained by ears as sound [35]. Eshach and Scwartz [11] (p. 751) showed that middle school students evidence similar ideas, as expressed in the following citation:

"In response to the question about two people talking ........... a few students drew pictures of bubbles that contained and carried sound from a person's mouth ...... One student answered: 'Sound is like bubbles of noise. Like small balls. Inside the balls there is a noise. When those balls open the sound comes out. That is how I understand it." "

\section{Expert examination of the instrument}

To establish face and content validity, seven experts were asked to review the instrument. These consisted of two physics professors teaching physics at a university level, two university science education lecturers, and three experienced high school physics teachers. This falls within the literature's rough consensus regarding the number of experts deemed sufficient for content validity. Yaghmaei [36] suggests three. Rubio, Berg-Weger, Tebb, Lee, and Rauch [37] recommend including three to ten experts. Chen [10] used six experts.

The seven experts were asked to review the first version of the questionnaire. More specifically they were asked to (a) complete the test, (b) associate each item with one of the instrument's objectives, (c) comment on the appropriateness of the objectives, (d) criticize the items, and (e) give a grade of 1 to 10 for each item in terms of its clarity. They were also invited to comment on the format and on how the phrasing of the statements could be improved. Then, all the experts and I met to discuss the comments and suggestions. Some items were modified or removed, and finally only items that were agreed upon by all of the experts as a result of the discussion were left in the final version.

\section{E. Statements' clarity}

After arriving at agreement on all the items, the final draft version was given to five middle school students, who were asked to read each of the questions and explain in their own words what they meant. The students were also asked to complete the questionnaire. Their comments were integrated into the questionnaire's final draft. These students were not included in the research sample. 


\section{F. Pilot study}

The draft versions of the test were administrated to 70 students from 2 schools. The results were used to modify several of the questions. As a result of this process three questions were completely removed from the questionnaire, as their item discrimination index was not satisfying. Individual item discrimination index statistics indicate how well the instrument distinguishes top scoring students from poorly performing students and should be greater than 0.3 [3]. In addition, 5 of the 70 students were interviewed and asked to repeat the questions in their own words as well as answer them. As a result of this process, another five items were slightly modified to further examine interesting patterns emerging from the preliminary data analysis.

The revised tests then underwent another session of expert examination until the experts agreed on the final version of the test.

\section{TESTING THE SCII}

\section{A. Participants}

The final draft of the test was distributed to 355 ninth grade students from across Israel; 170 students were male and the remaining 185 were female. About $30 \%$ of the students were Israeli Arabs, and $70 \%$ of them Israeli Jews; $80 \%$ of the students were from cities, while the remaining $20 \%$ were from villages or small towns. All of the schools included students from middle class socioeconomic status.

Sound is not part of the middle school science curriculum, but ninth graders do learn about electromagnetic radiation and that this radiation does not require any medium to pass through. To clarify this idea, teachers often compare the electromagnetic waves with sound waves and explain to the students that, unlike electromagnetic waves, sound waves do require a medium in order to travel from one place to another. Teachers also connect sound to changes in air pressure and density. A very popular experiment that teachers are familiar with and demonstrate to their students is that of putting a ringing clock inside a vacuum bell and showing the students that the ringing clock is not heard when air is taken out of the vacuum bell. Therefore, although sound is not directly included in the middle school science curriculum, students do learn the subject indirectly.

It took students between 40 and 60 minutes to complete the questionnaire.

\section{B. Individual item discrimination index}

The discrimination index for each of the assessment tool's statements with respect to the upper and lower $27 \%$ of the samples was calculated. One item, the discriminating power of which was still less than 0.3 in this form of the questionnaire, was discarded from the SCII. All the others' discrimination power was found to be in the range of $0.3-0.7$, ensuring the high quality of the assessment tool's statements.

\section{Internal reliability}

Each of the questionnaire's statements was associated with one of the predefined categories and their subcategories. These categories were based on conclusions drawn from a literature review, and from consultation with experts (see Sec. III A titled "Determining the SCII objectives or themes" above for details). Cronbach's alpha coefficient for dichotomous variables KR-20 was computed for the entire questionnaire, the two main objectives, and their subcategories. The results are presented in Table I.

TABLE I. Mean score and alpha Cronbach for the entire questionnaire and its subcategories.

\begin{tabular}{|c|c|c|c|}
\hline ISSUE & STATEMENTS & $\alpha$ & MEAN (SD) \\
\hline Entire questionnaire & 71 items & 0.906 & $27 \%(15.78 \%)$ \\
\hline Process properties & 23 items & 0.809 & $39.47 \%(22.88 \%)$ \\
\hline Connected to medium's particles movement & $1 b, 8 \mathrm{e}, 13 \mathrm{c}$ & 0.423 & $40.12 \%(23.34 \%)$ \\
\hline $\begin{array}{l}\text { Relates to changes in medium's pressure } \\
\text { or density }\end{array}$ & $\begin{array}{l}2 \mathrm{~d}, 3 \mathrm{a}, 4 \mathrm{c}, 5 \mathrm{~b}, 6 \mathrm{~d}, 7 \mathrm{~b}, 8 \mathrm{~d}, 9 \mathrm{e}, 10 \mathrm{~b}, 11 \mathrm{~d}, 12 \mathrm{c} \\
\quad 14 \mathrm{~b}, 15 \mathrm{a}, 16 \mathrm{c}, 17 \mathrm{c}, 18 \mathrm{a}, 19 \mathrm{c}, 19 \mathrm{~d}, 20 \mathrm{~b} \\
\quad 20 \mathrm{~d}\end{array}$ & 0.764 & $35.97 \%(32.22 \%)$ \\
\hline Materialistic properties & 48 items & 0.83 & $23.42 \%(15.87 \%)$ \\
\hline Invisible material & $3 \mathrm{c}, 3 \mathrm{~d}, 4 \mathrm{a}, 6 \mathrm{a}, 8 \mathrm{f}, 20 \mathrm{a}$ & 0.531 & $33.05 \%(25.02 \%)$ \\
\hline Corpuscular & $\begin{array}{l}\text { 1a,2a, 2b, 2c, } 4 b, 5 a, 6 c, 8 a, 8 b, 8 c, 10 a, \\
11 b, 12 a, 12 d, 12 e, 13 d, 15 b, 16 d, 19 a, 19 b\end{array}$ & 0.83 & $22.71 \%(20.00 \%)$ \\
\hline Pushable By objects & $2 \mathrm{a}, 2 \mathrm{~b}, 2 \mathrm{c}, 3 \mathrm{~b}, 5 \mathrm{a}, 6 \mathrm{c}, 8 \mathrm{a}, 11 \mathrm{~b}, 12 \mathrm{~d}, 15 \mathrm{~b}, 17 \mathrm{a}$ & 0.624 & $19.02 \%(18.87 \%)$ \\
\hline By medium & $3 \mathrm{~b}, 8 \mathrm{f}, 10 \mathrm{a}, 12 \mathrm{e}, 13 \mathrm{a}$ & 0.591 & $27.25 \%(26.75 \%)$ \\
\hline Frictional & $7 \mathrm{a}, 8 \mathrm{c}, 9 \mathrm{c}, 10 \mathrm{c}, 11 \mathrm{a}, 13 \mathrm{~d}, 14 \mathrm{a}, 18 \mathrm{~b}$ & 0.509 & $24.02 \%(19.26 \%)$ \\
\hline Containable & $4 a, 4 b, 6 a, 6 b, 8 f, 9 a, 9 d, 17 b$ & 0.636 & $33.00 \%(20.82 \%)$ \\
\hline Consumable & $11 \mathrm{c}, 16 \mathrm{e}$ & 0.327 & $22.96 \%(32.56 \%)$ \\
\hline Gravity sensitive & $13 b, 16 a, 16 b$ & 0.705 & $23.48 \%(33.63 \%)$ \\
\hline $\begin{array}{l}\text { Hearing is influenced by size or number } \\
\text { of sound particles }\end{array}$ & $1 \mathrm{a}, 2 \mathrm{~b}, 8 \mathrm{~b}, 8 \mathrm{c}, 12 \mathrm{a}, 12 \mathrm{~b}, 19 \mathrm{~b}$ & 0.708 & $26.95 \%(24.67 \%)$ \\
\hline Pass in vacuum & $9 \mathrm{~b}, 13 \mathrm{e}, 20 \mathrm{c}$ & 0.451 & $48.40 \%(31.12 \%)$ \\
\hline
\end{tabular}


As Table I shows, the alpha Cronbach of the entire questionnaire is pretty high, 0.96. The alpha Cronbach results of the process properties and material properties distractors are also quite satisfactory $(0.809$ and 0.83 , respectively). Overall students' percentage of correct answers to the entire questionnaire is $27 \%$.

The results also show that students associate sound with both material and process properties. Interestingly, the percentage of distractors indicating process properties that they marked as "correct" is higher than the percentage of distractors indicating materialistic properties that they marked as "incorrect" (39.47\% vs $23.42 \%)$. This means that that the students agreed with about $75 \%$ of the distractors that indicated that sound has material properties-reflecting a materialistic thinking of sound, and with about $40 \%$ of the distractors that indicated process properties-reflecting an association of sound with process. In addition, the range of the percentage of answers marked as "correct" for each of the process properties subcategories is between $35 \%$ and $41 \%$, and for answers marked as "incorrect" for each of the material properties subcategories the percentage ranges between $19 \%$ and $49 \%$. The lowest mean percentage of statements marked as "incorrect" in the material subcategories was for "pushable by object" $(19.02 \%)$. This means that for about $80 \%$ of the statements that students chose as correct that sound can be pushed by objects, and that students were certain of their response.

\section{DISCUSSION}

The purpose of this research was to develop the studentcentered Sound Concept Inventory Instrument, aiming at determining middle school students' conceptions of sound. Following Beichner [3], the assessment instrument development included the formulation of the assessment tool's objectives, constructing the instrument's items, and performing reliability and validity checks. A variety of sources were used, such as a literature review of students' concepts of sound, middle school students' responses to the first and second drafts of the questionnaire, physics textbooks, historical materials, and a panel of experts, including experienced physics teachers, physics professors, and science education professors with an extensive physics background. The experts were asked not only to examine the validity of the first draft of the test, but also to suggest other questions, or additional distractors for existing questions. This embracing of multiple sources is in line with Creswell [38], who suggests that we must "discuss plans to triangulate, or find convergence among sources of information, different investigators, or different methods of data collection" (p. 158) in order to increase the internal validity of an instrument. The use of the historical materials as a source was important and enriched the assessment tool. As can be seen from the testing of the instrument, students do possess concepts that existed through the history of sound concept development. This agrees with Galiliand Hazan [5] according to whom the history of science reveals the difficulties of today's students.

What emerged and was agreed upon by all the expertsafter reading the literature review on students' conception of sound and reviewing the historical development of the concept of sound, textbooks, and students' interviews-is that the majority of students' conception of sound can be divided into the following two main categories, as suggested by Chi [27]: (1) sound has material properties and (2) sound has process properties. The final form of the questionnaire includes 20 questions, composed of a total of 71 statements. Of those, 48 statements associate sound with materialistic properties, and 23 statements associate sound with processes.

The format used in the questionnaire allowed the students who completed it to decide whether the statements relating to the problems were true or false, and also to what degree they felt confidence in their answer on a 1-5 confidence scale. The confidence scale is very important, because a choice between true and false gives students a $50 \%$ chance of being correct, and without the scale we ran a great risk of counting guesses as correct answers based on relative certainty. To address this concern, only answers with a confidence level of 4 or 5 were used for the test analysis.

This was not a typical format for questionnaires. Usually the students have to choose the one option they believe best reflects the answer to the given question. The SSCI questionnaire can be used as a simple multiple choice one. However, adding the true or false element and the certainty scale provides additional information about students' perceptions, such as their associations of sound to both materialistic and process properties, even in the same problem.

The statistical results concerning the alpha Cronbach of the entire SCII questionnaire (71 items) is 0.906 , and the alpha Cronbach of its main subcategories is 0.809 for items that associate sound with process characteristics and 0.83 for items associating sound with materialistic characteristics. These statistical results are very satisfying, ensuring that the SCII is highly reliable. As to the process and material properties subcategories, their alpha Cronbach ranges between 0.327 and 0.83 . It is not surprising that some of these alpha Cronbach are smaller than 0.7, since each of these subcategories consists of only a few items.

The low alpha Cronbach of these subcategories may cast doubts upon the instrument's reliability, but it is worth noting in this context that the sound inventory was developed as an empirically based, student-centered instrument. Conventional concepts of validity and reliability cannot necessarily be applied to such empirically based developed instruments, because they emerge from a qualitative perspective [39-40], which stresses the trustworthiness and authenticity of data [41] rather than its consistency across constructs and measurements [10]. Many who have 
claimed this also argue that such instruments yield dependable results because the items originate from the respondent's point of view instead of from the researcher's presumption of reasonable answers, a process that also reduces the ambiguity of the items, and altogether yields high reliability.

The results from the sample used to test the instrument revealed that the average percentage of students' answers to statements that associate sound with material properties is significantly higher $(76.58 \%)$ than the average percentage of statements associating sound with process properties $(39.47 \%)$. A Wilcoxon signed ranks test revealed that this difference is statistically significant $(Z=10.56$, $p<0.001)$. A possible explanation for this result can be supported by Vosniaduo's framework theory [42] as follows. According to this theory, children start the knowledge acquisition process by organizing their everyday experiences into a naïve physics framework theory characterized by distinct ontological and epistemological commitments that are fundamentally different from current science. The materialistic thinking implies that the sound substance scheme may be considered as a framework theory; one may call it the material framework theory, yielded by children's organization of their everyday experiences. Indeed, even children as young as 4 years old possess the notion of substance conservation. Au, Siddle, and Rollins [43] found that children at this age understand that sugar dissolved in water continues to exist, even though it becomes invisible in the process.

Students try to explain different phenomena in light of the materialistic framework. In this process, the new scientific information is gradually added on to the existing but incompatible knowledge base, creating fragmentation, inconsistency, and synthetic models. This might be the reason that some of the students, in addition to the materialistic properties, though to a far lesser extent, expressed process properties as well. For instance, students might have heard about sound being a wave and that this wave is connected to air and pressure. This might lead them, in some situations, to decide that the statements that include the terms "pressure" and "air" are true, and furthermore to be confident in their decision. The different questions on the questionnaire may also have activated different students' ideas, based on their individual context. Regarding students' materialistic view, it is worth noting that the average percentages of statements associating sound with materialistic properties were high in all of the materialistic properties assessed by the instrument. It seems that middle school students view sound as a kind of invisible material, corpuscular in nature, which has size and weight, can be pushed, can be contained, can experience "drag" when moving in contact with some surface, and can move in a vacuum independently of the medium.

It is interesting to note that about half of the participants believe that sound can pass through a vacuum. According to Hrepic et al. [7], students' misconception that sound can propagate through vacuum strengthens the idea that students view sound as material because propagating through a vacuum indicates viewing sound as independent. However, in this study the average percentage of answers associating sound as being able to propagate through a vacuum was about 50\%, much lower than the average percentage to answers associating sound with other materialistic properties. This result might be explained by the fact that the experiment of a "clock in a vacuum" is quite typical and many middle schools make use of it. Students may therefore have discovered through such an experiment that sound cannot propagate through a vacuum. Knowing this one fact, however, does not lead these students to doubt their more general belief that sound is indeed material.

\section{CONCLUSION}

According to Engelhardt and Beichner [44] the widespread use of test instruments such as the Force Concept Inventory (FCI) [1] and the Test of Understanding Graphs in Kinematics [3] has brought in a new way of evaluating students' conceptual understanding. Engelhardt and Beichner further say that more instruments need to be developed in a variety of areas to allow instructors to better evaluate their students' understanding of physics concepts and to evaluate new teaching endeavors for their feasibility. In a sense, this paper is a response to their call, describing the development of a tool for assessing middle school students' understanding of sound, an area of physics for which no such tool previously existed.

The SCII assessment tool may contribute to three aspects of the field of science education: instruction, research, and methodology. Concerning the instructional aspect, it is worth noting that although the subject of sound should be an essential component of science curricula [11] and its understanding may contribute significantly to understanding both classical and modern physics [7], it does not receive the attention it deserves in school [11]. The existence of the SCII, which might be used as a largescale assessment tool at the state or national level, may contribute in changing this situation. Its results may lead to a discussion among physics educators on whether the physics of sound should be taught in more depth in schools and what topics should be included in the curriculum. Furthermore, it may be hoped that the SCII will help teachers modify their instruction to better address student difficulties with understanding sound.

From a research point of view, the existence of the SCII may help researchers to address a variety of research questions, such as whether there are gender differences in understanding the concept of sound. It could also help to assess novel learning environments, since many instruments like the SCII are used in pre- and post-instruction analysis [45]. The SCII may also encourage the 
development of such assessment tools for primary school, high school, and university students too.

From a methodological point of view, this paper provides a demonstration of how to build a student-centered assessment tool. In this regard the work of Rebello and Zollman [45] may come to mind. They have compared students' responses on four multiple-choice FCI questions with similar responses to equivalent open-ended questions. They found that a significant percentage of the open-ended responses fell into categories that are not included in the FCI multiple choices. When these alternative categories were presented to the students as distractors in a revised multiple-choice format, a significant percentage of the students chose these alternative responses. Rebello and Zollman warn us that using tools such as the FCI to evaluate the contribution of an intervention on understanding of the subject the assessment tool was designed for is problematic because "the effect of distracters could change during the course of instruction. The distracters that are effective before students have completed instruction may be ineffective or more effective after instruction." A student-centered questionnaire like the SCII, where students are encouraged to provide their own explanation, might help to improve the accuracy of such tools in pinpointing students' understanding of the subject they are designed to assess.

As to the limits of the SCII, it is worth noting that the participants in our study were from just one country. The results might be different if we used students from other countries, who might be exposed to physics at a level different from the one that the pool of students in this study were exposed to. Having said this, however, the SCII can be used to examine such cultural differences. It should be also stressed in concert with Engelhardt and Beichner [44] that, like any other assessment instrument, the SCII is not the end all be all of tests. It simply provides another data point for instructors and researchers to use to evaluate students' understanding. I agree with Engelhardt and Beichner [44] that "No one instrument or study can provide definitive answers. Data regarding students' understanding should be considered like evidence of validity-requiring several measurements through different means to arrive at the final answer." Therefore, the SCII should be used alongside other methods to provide credible and trustworthy assessments of students' understanding of sound.

\section{APPENDIX: QUESTIONNAIRE ON THE TOPIC OF SOUNDS}

Before you is a questionnaire about sound. It presents 27 phenomena. Each phenomenon comes with several statements. For each statement, mark "true" or "false." In addition, mark your level of certainty in the answer you have given from 1 (not sure at all) to 5 (very sure). Remember that each phenomenon can have more than one true statement.
1. Can there be a sound that we do not hear?

a. Yes. Our ears admit only sound particles of certain sizes. Animal ears admit different sizes of sound particles, so they can hear sounds we don't hear, and vice versa.

True/False. Certainty level in answer: 1, 2, 3, 4, 5

b. Yes. We can hear because our eardrum can detect changes in the movement of the air surrounding it. Our eardrum works in a certain range of air pressure.

True/False. Certainty level in answer: 1, 2, 3, 4,5

c. None of the above choices fits my basic viewpoint. My basic viewpoint is (please explain your viewpoint in the space provided below):

2. When we strum a guitar string, we hear a sound because:

a. The vibrating string releases sound particles and pushes them outward so they reach our ears. True/False. Certainty level in answer: 1, 2, 3, 4, 5

b. Each string releases and pushes outward sound particles of different sizes, and that's why they make different sounds.

True/False. Certainty level in answer: 1, 2, 3, 4, 5

c. The sound particles are actually in the air. The vibrating string pushes them. Because they are pushed with varying force, we hear different sounds.

True/False. Certainty level in answer: 1, 2, 3, 4,5

d. A vibrating string causes changes in the density and pressure of the air around it. This change travels to our ears and enable us to hear.

True/False. Certainty level in answer: 1, 2, 3, 4,5

e. None of the above choices fits my basic viewpoint. My basic viewpoint is (please explain your viewpoint in the space provided below):

3. Please refer to the following sound characteristics:

a. Sound is a moving change in air density (connected to air pressure).

True/False. Certainty level in answer: 1, 2, 3, 4,5

b. Sound moves because the air pushes it.

True/False. Certainty level in answer: 1, 2, 3, 4,5

c. Sound moves like an invisible liquid.

True/False. Certainty level in answer: 1, 2, 3, 4,5

d. Sound is not matter.

True/False. Certainty level in answer: 1, 2, 3, 4,5

e. None of the above choices fits my basic viewpoint. My basic viewpoint is: 
4. Large auditoriums have acoustic ceilings. The reason for this is:

a. The acoustic ceilings absorb the sounds made in the room like a sponge absorbs water.

True/False. Certainty level in answer: 1, 2, 3, 4, 5

b. The acoustic ceilings are made of a special net that allows the big sound particles to "catch" and stay in it.

True/False. Certainty level in answer: 1, 2, 3, 4, 5

c. The acoustic ceiling reduces the reflection of air density changes (sound), which is caused by sound sources in the room, from the ceiling back to the room space.

True/False. Certainty level in answer: 1, 2, 3, 4, 5

d. None of the above choices fits my basic viewpoint. My basic viewpoint is

5. Imagine that you're standing at the mouth of a very deep cave and you give a shout. An echo of your shout is heard. The reason for this is:

a. The sound particles coming out of your mouth collides with the sides of the cave like a tennis ball, and comes back to you after a time.

True/False. Certainty level in answer: 1, 2, 3, 4, 5

b. Changes in air pressure and density created by the shout return towards you from the walls of the cave after a time.

True/False. Certainty level in answer: 1, 2, 3, 4, 5

c. None of the above choices fits my basic viewpoint. My basic viewpoint is

6. Imagine the following two situations: in one you are standing on one end of a park, and your friend on the other. Your friend speaks to you. In the second situation imagine yourself on one end of a large, closed auditorium, with your friend on the other end. The park and the auditorium are of equal size. Your friend speaks to you, saying the precise same thing he had said in the park, at the same volume:

a. In the auditorium I will hear at a lower volume than in the park because the walls "absorb" the sound (like a sponge absorbs water).

True/False. Certainty level in answer: 1, 2, 3, 4, 5

b. In the auditorium I will hear better than in the park, because the sound has nowhere to "run" and it stays in the room.

True/False. Certainty level in answer: 1, 2, 3, 4, 5

c. I will hear louder in the room, because the sound particles colliding with the walls and roof return to the ears.
True/False. Certainty level in answer: 1, 2, 3, 4, 5

d. The change in densityand air pressure caused by your speaking friend return to the room's space and thus I will be better hear in the room (if there is no echo effect).

True/False. Certainty level in answer: 1, 2, 3, 4, 5

e. None of the above choices fits my basic viewpoint. My basic viewpoint is

7. A man is drilling a hole in the ground at the center of a large, empty park. Imagine that you are so far away from the man that you can barely hear the drill. Now, imagine that you are placing one of your ears to the ground, and close the other one. The sound of drilling:

a. Will not sound because the ground particles rub against the sound and "disrupt" its ability to pass.

True/False. Certainty level in answer: 1, 2, 3, 4, 5

b. Will be heard, because the change in density caused by the drill travels through the ground. True/False. Certainty level in answer: 1, 2, 3, 4, 5

c. None of the above choices fits my basic viewpoint. My basic viewpoint is

8. When we speak:

a. Our body releases sound particles that are pushed out by the vocal chords.

True/False. Certainty level in answer: 1, 2, 3, 4, 5

b. The size of the particles released is the reason for the difference between sounds.

True/False. Certainty level in answer: 1, 2, 3, 4, 5

c. When we shout, our throat hurts because more sound particles come out and rub against the sides of our throat.

True/False. Certainty level in answer: 1, 2, 3, 4, 5

d. We shake the air in our throats using our vocal chords. This shaken air makes a change in pressure that can travel distances. These pressure changes are, in effect, sound.

True/False. Certainty level in answer: 1, 2, 3, 4, 5

e. The speed in which the chords shake the air and cause changes in air's movement is related somehow to the different sounds created by them.

True/False. Certainty level in answer: 1, 2, 3, 4, 5

f. The sound coming out of our mouths is carried in invisible bubbles. These bubbles are pushed by 
the air, and when they reach the hearer's ears, the sound exits the bubbles and enters the ears. True/False. Certainty level in answer: 1, 2, 3, 4,5

g. None of the above choices fits my basic viewpoint. My basic viewpoint is

9. An alarm clock is placed inside a vacuum bell jar (a glass or plastic vessel from which all the air can be removed with a pump). The clock does not touch the walls and the base of the bell jar. A man standing outside the jar can hear the sound of the alarm clock. Now the air is removed from the bell jar. The man outside it:

a. Can't hear the alarm because the sound is trapped in the bell and can't escape.

True/False. Certainty level in answer: 1, 2, 3, 4,5

b. Will hear the alarm because its sound is unconnected to the presence of the air around it. True/False. Certainty level in answer: 1, 2, 3, 4,5

c. Will hear the alarm more loudly because now the air no longer rubs against the sound and "disrupts" its ability to move out.

True/False. Certainty level in answer: 1, 2, 3, 4,5

d. If we were to enter the bell jar, we would be able to hear the alarm (ignoring the fact that you will not be able to breathe).

True/False. Certainty level in answer: 1, 2, 3, 4,5

e. The sound of the alarm is very much diminished because there is no air to transmit the changes in air pressure (sound).

True/False. Certainty level in answer: 1, 2, 3, 4,5

f. None of the above choices fits my basic viewpoint. My basic viewpoint is

10. Please refer to the following statements regarding the propagation of sound in water:

a. In water, sound particles are pushed by water molecules.

True/False. Certainty level in answer: 1, 2, 3, 4,5

b. Sound can be heard in water because, like air, changes in density move from the source of the sound towards the hearer.

True/False. Certainty level in answer: 1, 2, 3, 4,5

c. In water, sound cannot be heard. The reason for this is that water is denser than air, and the water particles therefore rub against the sound and "disrupt" its movement.

True/False. Certainty level in answer: 1, 2, 3, 4,5 d. None of the above choices fits my basic viewpoint. My basic viewpoint is

11. Doctors use stethoscopes to listen to our lungs because:

a. The sound moves along the tubes of the stethoscope without interruption from friction with the air.

True/False. Certainty level in answer: 1, 2, 3, 4,5

b. The stethoscope prevents the sound particles from the lungs from colliding with sound particles from other sources.

True/False. Certainty level in answer: 1, 2, 3, 4,5

c. In the stethoscope, the sound is preserved within the tubes and does not dissipate.

True/False. Certainty level in answer: 1, 2, 3, 4,5

d. Sounds from inside the body vibrate the diaphragm (the side of the stethoscope attached to the body), creating air density changes within the tubes of the stethoscope which travel up the tubing to the doctor's ears.

True/False. Certainty level in answer: 1, 2, 3, 4,5

e. None of the above choices fits my basic viewpoint. My basic viewpoint is

12. We can hear sounds at different volumes: shouts and whispers. The reason for this is:

a. When we speak loudly we release more sound particles which also hurt our throat.

True/False. Certainty level in answer: 1, 2, 3, 4,5

b. When we speak loudly we release bigger sound particles which also hurt our throats.

True/False. Certainty level in answer: 1, 2, 3, 4,5

c. When we speak loudly the air's pressure levels generated near our mouth are greater.

True/False. Certainty level in answer: 1, 2, 3, 4,5

d. When we speak loudly we push the sound particles faster.

True/False. Certainty level in answer: 1, 2, 3, 4,5

e. When we speak loudly we push the air with more force. The air pushes the sound particles faster.

True/False. Certainty level in answer: 1, 2, 3, 4,5

f. None of the above choices fits my basic viewpoint. My basic viewpoint is

13. Imagine you are on the moon, and there's an explosion close by (there is no air on the moon). 
a. I will hear the sound of the explosion, but the sound will be softer because there's no air to push the sound to my ears.

True/False. Certainty level in answer: 1, 2, 3, 4, 5

b. I will hear the sound of the explosion, but it will be softer because gravity on the moon is lighter and the sound will disperse.

True/False. Certainty level in answer: 1, 2, 3, 4, 5

c. Because there's no air on the moon to be moved, it is impossible to generate soundson its space.

True/False. Certainty level in answer: 1, 2, 3, 4, 5

d. I will hear the explosion louder than on Earth because in space there are no air molecules to rub against the sound particles and disrupt their progress.

True/False. Certainty level in answer: 1, 2, 3, 4, 5

e. In terms of sound transmission, there is no difference between the moon and the Earth, and I will therefore hear the explosion at the same volume I would on Earth.

True/False. Certainty level in answer: 1, 2, 3, 4, 5

f. None of the above choices fits my basic viewpoint. My basic viewpoint is

14. Imagine that several long, thin tables have been placed before you. They are made of different materials: plastic, wood, iron, Styrofoam, etc. Now you place your ear at one end of a table ( $a$ different one each time) and someone hits the other end.

a. On the Styrofoam table the sound will be heard because the styrofoam's density is low and there's only small friction between the sound and the Styrofoam particles.

True/False. Certainty level in answer: 1, 2, 3, 4, 5

b. On the wooden table the sound will be heard because the wood is dense and density change (sound) in the wood can travel from one edge to another.

True/False. Certainty level in answer: 1, 2, 3, 4, 5

c. None of the above choices fits my basic viewpoint. My basic viewpoint is

15. Imagine that you've blown up a balloon and put it to your ear. Imagine that your friend places his mouth to the balloon and speaks to you through it. Will you hear your friend?

a. Yes. Because the changes in pressure created by your speaking friend travel through the balloon.
True/False. Certainty level in answer: 1, 2, 3, 4, 5

b. No. Because the balloon blocks the passage of the sound particles that collide with it.

True/False. Certainty level in answer: 1, 2, 3, 4, 5

c. None of the above choices fits my basic viewpoint. My basic viewpoint is

16. A trumpet player is playing in the town square. Imagine that you are nearby, and can hear the sound of the trumpet clearly. Now, you move away from the trumpet player as he keeps playing. The sound of the trumpet will be heard to you weaker because:

a. Gravity pulls the sound downward, and as a result it does not reach my ears.

True/False. Certainty level in answer: 1, 2, 3, 4, 5

b. Sound rises, because it's lighter than air, and as a result it does not reach my ear.

True/False. Certainty level in answer: 1, 2, 3, 4, 5

c. As I move farther away, the intensity of the changes in air pressure reaching my ears goes weaker (i.e. the changes in air density are smaller).

True/False. Certainty level in answer: 1, 2, 3, 4, 5

d. As I move away from the trumpet, the sound particles get farther away from each other, and fewer particles enter my ear.

True/False. Certainty level in answer: 1, 2, 3, 4, 5

e. As I move away from the trumpet, the sound of the trumpet runs out.

True/False. Certainty level in answer: 1, 2, 3, 4,5

f. None of the above choices fits my basic viewpoint. My basic viewpoint is

17. Imagine that sounds are made inside a room. If you shut the door, the sound heard on its other side will hardly be heard. This is because:

a. A significant part of the sound particles are rebuffed (like a ball) by the walls and the door. True/False. Certainty level in answer: 1, 2, 3, 4, 5

b. A significant part of the sound is "absorbed" (like water in a sponge) in the walls and door and thus most of it does not travel out.

True/False. Certainty level in answer: 1, 2, 3, 4, 5

c. The walls and door significantly prevent the transmission of changes in air pressure from inside the room to its outside.

True/False. Certainty level in answer: 1, 2, 3, 4, 5

d. None of the above choices fits my basic viewpoint. My basic viewpoint is (please explain your viewpoint in the space provided below): 
18. A boat's motor can be either in or out of the water. Imagine that you're at sea, far from a boat with its motor in the water. While your head is out of the water, you can barely hear the motor. Now, imagine that you put your ear in the water.

a. You will hear the motor because changes in the water's density propagate through the water. True/False. Certainty level in answer: 1, 2, 3, 4, 5

b. The motor will not sound, because the friction of the water will "disrupt" the sound's ability to move.

True/False. Certainty level in answer: 1, 2, 3, 4, 5

c. None of the above choices fits my basic viewpoint. My basic viewpoint is (please explain your viewpoint in the space provided below):

19. When you stand behind the door to a room in which music is playing, you can still hear the music because:

a. The sound is made of small particles that can pass through gaps, like the one between the door and the floor.

True/False. Certainty level in answer: 1, 2, 3, 4,5

b. The sound is made of different sized particles. The smallest ones can get through doors and walls that are not totally sealed.

True/False. Certainty level in answer: 1, 2, 3, 4, 5

c. The changes in air density formed in the gap between the door and the floor travel outside. True/False. Certainty level in answer: 1, 2, 3, 4, 5

d. The sounds in the room cause the wall to vibrate. The vibrating wall causes the air on the other side to vibrate and slightly changes the air pressure there.

True/False. Certainty level in answer: 1, 2, 3, 4, 5

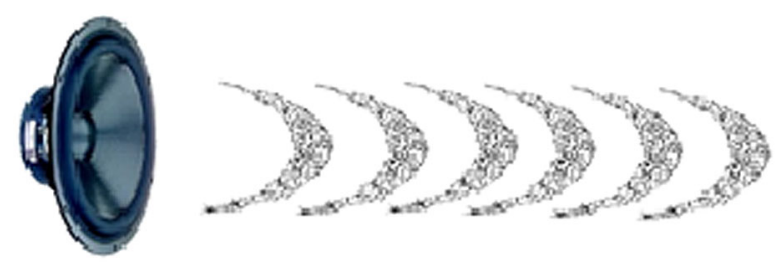

FIG. 1 (color online). An illustration of a "loudspeaker" and curves for question $20 \mathrm{~d}$.

e. None of the above choices fits my basic viewpoint. My basic viewpoint is (please explain your viewpoint in the space provided below):

20. Please refer to the following characteristics regarding sound:

a. Sound is invisible matter.

True/False. Certainty level in answer: 1, 2, 3, 4, 5

b. Sound is created by changes in the density of a matter that fills a space (medium). The change in the medium's density propagates through it. True/False. Certainty level in answer: 1, 2, 3, 4, 5

c. Sound can also travel through vacuum (a place without matter)

True/False. Certainty level in answer: 1, 2, 3, 4, 5

d. The curves in Fig. 1 may represent the change in the density of the air moving from the loudspeaker, creating the sounds we hear. True/False. Certainty level in answer: 1, 2, 3, 4, 5

e. None of the above choices fits my basic viewpoint. My basic viewpoint is (please explain your viewpoint in the space provided below):
[1] D. Hestenes, M. Wells, and G. Swackhamer, Force concept inventory, Phys. Teach. 30, 141 (1992).

[2] D. Hestenes and M. Wells, A mechanics baseline test, Phys. Teach. 30, 159 (1992).

[3] R. Beichner, Testing student interpretation of kinematics graphs, Am. J. Phys. 62, 750 (1994).

[4] R. Trumper, University students' conceptions of basic astronomy concepts, Phys. Educ. 35, 9 (2000).

[5] I. Galili and A. Hazan, Learners' knowledge in optics: Interpretation, structure and analysis, Int. J. Sci. Educ. 22, 57 (2000).

[6] S. Vosniadou, C. Ioannides, A. Dimitrakopoulou, and E. Papademetriou, Designing learning environments to promote conceptual change in science, Learn. Instr. 11, 381 (2001).

[7] Z. Hrepic, D. A. Zollman, and N. S. Rebello, Identifying students' mental models of sound propagation: The role of conceptual blending in understanding conceptual change, Phys. Rev. ST Phys. Educ. Res., 6, 020114 (2010).

[8] R. E. Mayer, in Reconsidering Conceptual Change: Issues in Theory and Practice, edited by M. Limon and L. Mason (Kluwer Academic Publishers, Dordrecht, Netherlands, 2002), pp. 101-111.

[9] R. Good, N. Lederman, J. Gess-Newsome, W. McComas, and C. Cummins, A symposium and paper presented at the annual international meeting of the Association for the 
Education of Teachers in Science, Akron, OH, 2000 (unpublished).

[10] S. Chen, Development of an instrument to assess views on nature of science and attitudes toward teaching science, Sci. Educ. 90, 803 (2006).

[11] H. Eshach and J. L. Schwartz, Sound stuff? Naive materialism in middle-school students' conceptions of sound, Int. J. Sci. Educ. 28, 733 (2006).

[12] R. Trumper, A cross-college age study of science and nonscience students' conceptions of basic astronomy concepts in preservice training for high-school teachers, J. Sci. Educ. Technol. 10, 189 (2001).

[13] D. Layton, Science for the People (George Allen \&Unwin Ltd., London, 1973).

[14] F. Abd-El-Khalick, R. L. Bell, and N. G. Lederman, The nature of science and instructional practice: Making the unnatural natural, Sci. Educ. 82, 417 (1998).

[15] N. G. Lederman, F. Abd-El-Khalick, B. L. Bell, and R. S. Schwartz, Views of nature of science questionnaire: Toward valid and meaningful assessment of learners' conceptions of nature of science, J. Res. Sci. Teach.39, 497 (2002).

[16] N. G. Lederman and M. O'Malley, Students' perceptions of tentativeness in science: Development, use, and sources of change, Sci. Educ. 74, 225 (1990).

[17] J. Clement, in Handbook of Research Methodologies for Science and Mathematics Education, edited by R. Lesh and A. Kelly (Lawrence Erlbaum, Hillsdale, NJ, 2000), pp. 341-385.

[18] R. S. Schwartz, N. G. Lederman, and B. A. Crawford, Developing views of nature of science in an authentic context: An explicit approach to bridging the gap between nature of science and scientific inquiry, Sci. Educ. 88, 610 (2004).

[19] N. Dogan and F. Abd-El-Khalick, Turkish grade 10 students' and science teachers' conceptions of the nature of science: A national study, J. Res. Sci. Teach. 45, 1083 (2008).

[20] P. Tamir, An alternative approach to the construction of multiple-choice test items, J. Biol. Educ. 5, 305 (1971).

[21] P. Marmaroti and D. Galanopoulou, Pupils' understanding of photosynthesis. A questionnaire for the simultaneous assessment of all aspects, Int. J. Sci. Educ. 28, 383 (2006).

[22] A. Ryan and G. Aikenhead, Students' preconceptions about the epistemology of science, Sci. Educ., 76, 559 (1992).

[23] I. Galili, Promotion of cultural content knowledge through the use of the history and philosophy of science, Sci. Educ. 21, 1283 (2012).

[24] J. Lautrey and K. Mazens, Is children's naive knowledge consistent? A comparison of the concepts of sound and heat, Learn. Instr. 14, 399 (2004).

[25] E. Boyes and M. Stanisstreet, Development of pupils' ideas about seeing and hearing-The path of light and sound, Res. Sci. Technol. Educ. 9, 223 (1991).

[26] H. M. Asoko, J. Leach, and P. H. Scott, Proceedings of the Annual Conference of the British Educational Research Association, Roehampton Institute, London, 1991 (unpublished).
[27] M. T. H. Chi, in Cognitive Models of Science: Minnesota Studies in the Philosophy of Science edited by R. Giere (University of Minnesota Press, Minneapolis, MN, 1992).

[28] M. T. H. Chi and J. D. Slotta, The ontological coherence of intuitive physics, Cognit. Instr. 10, 249 (1993).

[29] M. T. H. Chi, J. D. Slotta, and N. A. de Leeuw, From things to processes: A theory of conceptual change for learning science concepts, Learn. Instr. 4, 27 (1994).

[30] M. Reiner, J. D. Slotta, M. T. H. Chi, and L. B. Resnick, Naïve physics reasoning: A commitment to substancebased conceptions, Cognit. Instr. 18, 1 (2000).

[31] R. Driver, A. Squires, P. Rushworth, and V. WoodRobinson, Making Sense of Secondary Science (Routledge, London, England, 1994).

[32] D. Watt and T. Russell, Sound, Pimary SPACE Project Research Report (Liverpool University Press, Liverpool, England, 1990).

[33] Lucretius, On the Nature of the Universe (translated by R. E. Latham) (Penguin Books, Harmondsworth, England, 1951).

[34] R. J. Roedel, S. El-Ghazaly, T. R. Rhoads, and E. ElSharawy, in Proceedings of Frontiers in Education Conference, Tempe, AZ, 1998 (unpublished).

[35] I. S. Caleon and R. Subramaniam, From Pythagoras to Sauveur: Tracing the history of ideas about the nature of sound, Phys. Educ. 42, 173 (2007).

[36] F. Yaghmaei, Content validity and its estimation, J. Gen. Internal Medicine 3, 25 (2003).

[37] D. M. Rubio, M. Berg-Weger, S. S. Tebri, E. S. Lee, and S. Rauch, Objectifying content validity: Conducting a content validity study in social work research, Social Work Research 27, 94 (2003).

[38] J. W. Creswell, Research Design: Qualitative and Quantitative Approaches (SAGE Publications, Thousand Oaks, CA, 1994).

[39] G. Aikenhead and A. Ryan, The development of a new instrument: Views on Science-Technology-Society (VOSTS), Sci. Educ., 76, 477 (1992).

[40] P. A. Rubba, C. Schoneweg Bradford, and W. J. Harkness, A new scoring procedure for the Views on Science Technology-Society instrument, Int. J. Sci. Educ., 18, 387 (1996).

[41] D. A. Erlandson, E. L. Harris, B. L. Skipper, and S. D. Allen, Doing Naturalistic Inquiry (SAGE Publications, Newbury Park, CA, 1993).

[42] S. Vosniadou and W. F. Brewer, Mental models of the day/ night cycle, Cogn. Sci. 18, 123 (1994).

[43] T. K. Au, A. L. Siddle, and K. B. Rollins, Developing an intuitive understanding of conservation and contamination: Invisible particles as a plausible mechanism, Dev. Psychol. 29, 286 (1993).

[44] P. V. Engelhardt and R. J. Beichner, Students' understanding of direct current circuits, Am. J. Phys. 72, 98 (2004).

[45] N. S. Rebello and D. A. Zollman, The effect of distracters on student performance on the force concept inventory, Am. J. Phys. 72, 116 (2004). 